\title{
Association between circulating zinc-a2-glycoprotein levels and the different phenotypes of polycystic ovary syndrome
}

\author{
Chenchen Tang ${ }^{1)}$, Xiaoqiang Li $^{2}$, Shiguo Tang ${ }^{1)}$, Yi Wang ${ }^{1)}$ and Xingrong Tan ${ }^{1)}$ \\ 1) Department of Endocrinology, 9th People's Hospital of Chongqing, Beibei City, Chongqong, 400700, China \\ 2) Department of Clinical Laboratory, Children's Hospital of Chongqing Medical University, Yuzhong City, Chongqing 400014, China
}

\begin{abstract}
Polycystic ovary syndrome (PCOS) diagnosis combines various clinical phenotypes. The definition of PCOS is still controversial because insulin resistance (IR) and dysmetabolism do not constitute PCOS diagnostic criteria. We analyzed whether a circulating biomarker zinc- $\alpha 2$-glycoprotein (ZAG) related to IR and metabolic dysfunction can predict PCOS phenotypes. We then recruited 100 PCOS patients and 99 healthy women as the control group to assess the relationship between ZAG and metabolic characteristics. The euglycemic-hyperinsulinemic clamp helped assess insulin sensitivity, and the enzyme immunometric assay was deployed for ZAG levels. Our PCOS cohort presented sixty-nine patients with hyperandrogenism, eighty-six patients with chronic oligoanovulation, and eighty-one patients with polycystic ovaries by ultrasonographic evaluation. Additionally, the circulating ZAG levels were considerably reduced in all PCOS patients compared with healthy women $(p<0.05$ or $p<0.01)$. Additionally, sixty-nine PCOS patients had IR, and circulating ZAG levels were also different among the phenotypes. Furthermore, the normoandrogenic type specifically exhibited the highest circulating ZAG levels among all PCOS phenotypes $(p<0.05$ or $p<0.01)$. Additionally, normoandrogenic phenotype patients had reduced HOMA-IR scores and greater M-values than those in the classic phenotypes $(p<0.05)$. The circulating ZAG levels, however, were not associated with oligoanovulation but were correlated with hyperandrogenism and PCO morphology. In summary, circulating ZAG levels serve as suitable PCOS phenotype biomarkers, aiding physicians to identify women who merit screening.
\end{abstract}

Key words: Polycystic ovary syndrome (PCOS), Phenotype, Zinc- $\alpha 2$-glycoprotein (ZAG), Insulin resistance (IR)

POLYCYSTIC OVARY SYNDROME (PCOS), also known as metabolic reproductive syndrome (MRS), is a common condition in reproductive-age women. Many

Submitted Nov. 19, 2018; Accepted Oct. 22, 2019 as EJ18-0506

Released online in J-STAGE as advance publication Nov. 21, 2019

Correspondence to: Xingrong Tan, Department of Endocrinology, 9th People's Hospital of Chongqing, No. 9 Hejia Rd, Beibei City, Chongqong, 400700, China.

E-mail: 447929013@qq.com

Correspondence to: Yi Wang, Department of Endocrinology, 9th People's Hospital of Chongqing, No. 9 Hejia Rd, Beibei City, Chongqong, 400700, China.

E-mail: wangyi-med@sohu.com

Abbreviations: PCOS, polycystic ovary syndrome; TC, total cholesterol; BMI, body mass index; HDL-C, high-density lipoprotein cholesterol; WC, waist circumference; TG, triglyceride; FAT (\%), body fat $\%$; LDL-C, Low-density lipoprotein cholesterol; SBP, systolic blood pressure; FFA, free fatty acid; DBP, diastolic blood pressure; SHBG, sex hormone-binding globulin; FBG, fasting blood glucose; LH, luteinizing hormone; $2 \mathrm{~h}-\mathrm{BG}, 2 \mathrm{~h}$ post glucose load blood glucose; FSH, follicular stimulating hormone; FIns, fasting plasma insulin; FAI, free androgen index; 2 h-Ins, $2 \mathrm{~h}$ plasma insulin after glucose overload; Test, testosterone clinical consequences, such as infertility, obesity and impaired glucose tolerance, are observed in PCOS patients. In addition, these women have an increased risk of developing cardiovascular disease and type 2 diabetes mellitus (T2DM) [1, 2].

Although insulin resistance (IR) is not included in the criteria for PCOS, this condition is a common physiological abnormality in PCOS patients with multiplemetabolic dysfunctions [3]. IR plays a crucial role in the onset of PCOS [4]. Approximately $70 \%$ of PCOS women have IR $[5,6]$. However, the true prevalence of IR individuals with PCOS is unclear because of limitations, such as IR assessment methods. Thus, it is important to identify reliable biomarkers for predicting IR using different features or phenotypes than those used for diagnosing PCOS.

A number of adipokines are produced by tissues, such as adiponectin (ADIPOQ), zinc- $\alpha_{2}$-glycoprotein (ZAG) and leptin. These adipokines regulate glucose and lipid metabolism and insulin action and play important roles in the pathogenesis of IR, obesity, T2DM and cardiovas- 
cular diseases [7-9]. In a previous study, ZAG was shown to promote lipolysis in vitro and decrease body weight by increasing fat loss and decreasing triglyceride levels (TG) and other components of metabolic syndrome (MetS) [10]. In another study, ZAG mRNA and protein levels were observed to be downregulated in the adipose tissue of obese individuals with IR relative to lean subjects [11]. Furthermore, circulating ZAG levels are low in impaired glucose tolerance (IGT) subjects and newly diagnosed T2DM patients and have been positively correlated with ADIPOQ but inversely correlated with body mass index (BMI), waist-to-hip ratio (WHR), levels of TG, fasting insulin (FIns), HbA1c, and homeostasis model assessment of insulin resistance (HOMAIR) scores [12]. In addition, other studies have shown that low circulating ZAG levels are associated with IR. The administration of sitagliptin, a dipeptidyl peptidaseIV (DPP-IV) inhibitor, significantly increases plasma ZAG concentrations following improved IR in T2DM patients, further demonstrating the relationships between ZAG and IR [13]. Importantly, Lai et al. observed that circulating ZAG levels were much lower in PCOS patients than in healthy women [14]. However, no studies have demonstrated a relationship between circulating ZAG levels and the different features or phenotypes used for diagnosing PCOS. Therefore, in the current study, we investigated whether the different features and phenotypes used to diagnose PCOS, alone or in combination, are related to circulating ZAG levels in PCOS patients. To address this issue, 199 women, including 100 PCOS patients and 99 healthy women, were recruited in this study. To assess the relationship between circulating ZAG levels and clinical phenotypes in PCOS patients, we will use state-of-the-art methods.

\section{Materials and Methods}

\section{Study population}

One hundred ninety-nine Chinese women, including 100 PCOS patients and 99 healthy women, participated in the current study. All PCOS patients had been referred to the Department of Endocrinology or Gynecology of the 9th People's Hospital of Chongqing from January 2015 to January 2018 due to menstrual irregularities, anovulation and or hyperandrogenism. According to the 2003 Rotterdam consensus criteria (the Rotterdam ESHRE/ASRM-sponsored PCOS consensus workshop group) [15], PCOS was diagnosed in these women by the presence of at least two of the following three features: 1) oligo-amenorrhea or chronic anovulation; 2) clinical and/or biochemical hyperandrogenism; and 3) the appearance of polycystic ovaries by ultrasonographic evaluation after the exclusion of other known causes of hyperandrogenemia and ovulatory dysfunction, including 21-hydroxylase deficiency, congenital adrenal hyperplasia, Cushing syndrome, androgen-secreting tumors, thyroid disease, and hyperprolactinemia. The normoandrogenic phenotype of PCOS was diagnosed by the presence of two of the following features without hyperandrogenemia: 1) oligo-amenorrhea or chronic anovulation; and 2) ultrasound appearance of polycystic ovaries. The ovulatory phenotype of PCOS was diagnosed by 1) clinical and/or biochemical hyperandrogenism and 2) the ultrasonographic appearance of polycystic ovaries. The no-PCOS morphology of PCOS was diagnosed by 1) oligo-amenorrhea or chronic anovulation and 2) clinical and or biochemical hyperandrogenism.

The diagnosis of metabolic syndrome was based on the 2009 Joint Interim Statement of the IDF. The inclusion criteria were 16- to 39-year-old women with newly diagnosed PCOS, while the exclusion criteria included T2DM or T1DM patients, women with other diseases and women who had used drugs [16]. Ninety-nine healthy women with regular menstrual periods were recruited as the control group from the community or schools through advertisements or routine medical check-ups. None of the healthy women had used any drugs within the previous 6 months. All of the individuals provided written informed consent before attending this study, which was performed according to the Declaration of Helsinki and was registered by the Chinese Clinical Trial Registry (No. ChiCTR-OOC-14005314).

\section{Oral glucose tolerance test (OGTT) and euglycemic- hyperinsulinemic clamp (EHC)}

An OGTT was performed for each of the 100 PCOS patients and the 99 healthy women. At 7:00 in the morning on the study days, after a 12-h overnight fast, all individuals were administered $75 \mathrm{~g}$ glucose, and venous blood was subsequently drawn at the indicated times $(0$, 30,60 , and $120 \mathrm{~min}$ ) to measure glucose and insulin levels.

EHCs were performed on all 100 PCOS patients and 99 healthy women, as previously described [17, 18]. During the EHC, regular human insulin $(1 \mathrm{mU} / \mathrm{kg} / \mathrm{min})$ was infused for $2 \mathrm{~h}$, and a variable infusion of $20 \%$ glucose was administered to maintain plasma glucose at the fasting level. The glucose disposal rate (GDR) was defined as the rate of glucose infusion (GIR) during the stable period of the EHC. The M-value was calculated according to the equation $\mathrm{M}$-value $=\mathrm{GIR}-\mathrm{SC}$, where $\mathrm{SC}$ is the space correction, and all values were computed in dimensions of $\mathrm{mg} /(\mathrm{kg} \cdot \mathrm{min})$ [19]. Blood samples for ZAG and insulin measurements were obtained at the indicated times, immediately cooled, centrifuged, subsequently used to prepare serum and stored at $-80^{\circ} \mathrm{C}$ until 
use.

\section{Anthropometric measurements}

BMI was calculated as weight divided by height squared. Bioelectricalimpedance (BIA-101; RJL Systems, China) was used to measure body fat (FAT \%). The HOMA-IR scores were calculated as previously reported [20]: HOMA-IR $=$ FIns $(\mathrm{mU} / \mathrm{mL}) \times$ fasting blood glucose $(\mathrm{FBG}, \mathrm{mmol} / \mathrm{L}) / 22.5$. Blood glucose levels were measured using the glucose-oxidase method and by anion-exchange HPLC. Insulin was measured by chemiluminescence. Free fatty acids (FFAs), totalcholesterol (TC), high-density lipoprotein cholesterol (HDL-C), low-density lipoprotein (LDL-C) and TG were measured as previously described [12].

\section{Measurements of circulating ZAG and other hormones}

Serum ZAG concentrations were determined by ELISA kits (sigma, RAB1779) according to the manufacturer's protocol. The detection limit was $0.04 \mathrm{ng} / \mathrm{mL}$, and the intra- and inter-assay variations were 2.56 and $6.63 \%$, respectively. The serum levels of hormones, including luteinizing hormone ( $\mathrm{LH})$, follicle-stimulating hormone (FSH) and testosterone (Test), were examined by an electrochemiluminescence assay (Roche Diagnostics $\mathrm{GmbH}$ ). Serum testosterone was measured by RIA. The level of sex hormone binding globulin (SHBG) was measured using an automated analyzer. The free androgen index (FAI) was calculated using the following formula: $\mathrm{FAI}=($ Test $/ \mathrm{SHBG}) \times 100$.

\section{Statistical analysis}

All data were analyzed using SPSS 22.0 (Chicago, IL) and the results were expressed as the means $\pm \mathrm{SD}$ or median (interquartile range). Abnormally distributed data were log-transformed prior to analysis. Comparisons among groups were performed by ANOVA, unpaired $t$ test, or paired $t$-test. Correlations between variables were assessed using partial correlation analyses by controlling for the covariates. The associations among ZAG and other anthropometric variables and hormones were assessed by multiple regression analyses. Logistic regression analyses were performed to assess the associations between the dichotomized ZAG variable, as a dependent categorical variable, and either the PCOS diagnostic features or the PCOS phenotypes, as independent variables.

\section{Results}

Among the 100 PCOS patients recruited in this study, sixty-nine patients exhibited hyperandrogenism, eighty- six patients exhibited chronic oligo- or anovulation, and eighty-one patients exhibited polycystic ovaries by ultrasonographic evaluation. Thirty-six patients were diagnosed with the classic phenotype according to the 2003 Rotterdam consensus criteria, and fourteen patients with ovulation were considered to be the ovulatory phenotype. Another thirty-one patients with normal androgen levels were reported as the normoandrogenic phenotype, while the remaining nineteen patients without ovarian polycystic changes were reported as the no-PCO morphology phenotype. The clinical features and hormone levels in PCOS patients and healthy women are shown in Table 1. Patients with the normoandrogenic phenotype were leaner (lower BMI and waist circumference) and had lower fasting insulin, 2-h plasma insulin after glucose overload (2h-Ins), FAT\%, Test, and FAI levels as well as a higher M-value, and higher FSH and SHBG levels compared with the classic phenotype $(p<0.05, p<$ $0.01)$. In addition, the ovulatory phenotype had higher TG and FSH levels relative to the classic phenotype ( $p<$ $0.05)$. The no-PCO morphology phenotype had higher levels of LDL-C than the classic phenotype $(p<0.01)$. In all PCOS phenotypes, compared with the healthy controls, the M-values were significantly decreased, whereas the HOMA-IR scores were increased $(p<0.05)$, showing that most of the patients were IR. In fact, sixty-nine PCOS patients had IR. The insulin resistance was highest in the ovulatory phenotype and lowest in the normoandrogen phenotype (Table 1). As shown in Table 1, the PCOS patients had lower circulating ZAG levels than the healthy controls $(p<0.05)$, and the highest ZAG levels were observed in the normoandrogenic phenotype among the three PCOS phenotypes $(p<0.05, p<0.01)$.

When the relationship between circulating ZAG levels and the phenotype of PCOS was examined (Table 2, model 1), circulating ZAG levels were significantly associated with hyperandrogenism and polycystic ovary morphology $(p<0.01)$, but not oligoanovulation. However, when M-values and HOMA-IR scores were included in the analysis (Table 2, model 2), the levels of circulating ZAG were observed to be associated with hyperandrogenism and M-values, suggesting that circulating ZAG levels were associated with IR and hyperandrogenism.

We next further categorized PCOS individuals into different phenotypes and analyzed the relationships between these phenotypes and circulating ZAG levels. We observed that circulating ZAG levels were significantly associated with each phenotype of PCOS $(p<$ 0.001) (Table 3). When HOMA-IR scores and M-values were included in the analysis, these associations were unaffected ( $p<0.001)$ (Table 3). However, when the Mvalues and HOME-IR scores were included in the multivariable analysis, the classic and no-PCO morphology 
Table 1 Main characteristics in the study population.

\begin{tabular}{|c|c|c|c|c|c|}
\hline Variable & $\begin{array}{l}\text { Controls } \\
(n=99)\end{array}$ & $\begin{array}{l}\text { Classic } \\
(n=36)\end{array}$ & $\begin{array}{l}\text { Normoandrogenic } \\
\quad(n=31)\end{array}$ & $\begin{array}{l}\text { Ovulatory } \\
(n=14)\end{array}$ & $\begin{array}{l}\text { no-PCO morphology } \\
\qquad(n=19)\end{array}$ \\
\hline Age (year) & $25(24-27)$ & $25.7 \pm 4.4$ & $25.8 \pm 4.7$ & $25.1 \pm 3.4$ & $26.6 \pm 4.5$ \\
\hline BMI $\left(\mathrm{kg} / \mathrm{m}^{2}\right)$ & $20(18.59-21.33)$ & $25.5 \pm 4.0^{* *}$ & $23.1 \pm 4.3 * * \mathbf{\Lambda} \boldsymbol{\Delta} \#$ & $28.1 \pm 5.1 * *$ & $24.7 \pm 4.9 * *$ \\
\hline $\mathrm{WC}(\mathrm{cm})$ & $70.2 \pm 7.4$ & $83.4 \pm 9.8 * *$ & $78.3 \pm 10.4 * * \boldsymbol{\Lambda}$ & $87.5 \pm 14.1 * *$ & $83.2 \pm 11.4 * *$ \\
\hline FAT $\%$ & $26.7 \pm 5.6$ & $37.3 \pm 8.0^{* *}$ & $32.8 \pm 8.5^{* * \boldsymbol{\Lambda}}$ & $39.2 \pm 13.9 * *$ & $35.0 \pm 8.8 * *$ \\
\hline $\mathrm{SBP}(\mathrm{mmHg})$ & $109.0 \pm 8.1$ & $115.7 \pm 9.6^{* *}$ & $115.2 \pm 11.1 * *$ & $118.3 \pm 13.0^{* *}$ & $116.0 \pm 10.7^{* *}$ \\
\hline DBP (mmHg) & $74.8 \pm 7.8$ & $76.0 \pm 5.6$ & $76.0 \pm 6.6$ & $79.6 \pm 10.5^{*}$ & $80.1 \pm 8.20 * *$ \\
\hline FBG (mmol/L) & $4.45 \pm 0.46$ & $4.92 \pm 0.66^{* *}$ & $4.87 \pm 0.37 * *$ & $5.11 \pm 0.68 * *$ & $4.88(4.56-5.32)^{* *}$ \\
\hline $2 \mathrm{~h}-\mathrm{BG}(\mathrm{mmol} / \mathrm{L})$ & $5.39 \pm 1.12$ & $7.20 \pm 1.80 * *$ & $6.68 \pm 1.98 * *$ & $8.54 \pm 1.62 * *$ & $7.70 \pm 2.05 * *$ \\
\hline FIns (mU/L) & $7(6.1-8.7)$ & $19.0 \pm 14.0 * *$ & $13.3 \pm 8.2 * * \mathbf{\Delta} \mathbf{\Delta} \#$ & $21.0 \pm 8.5^{* *}$ & $21.0 \pm 15.1 * *$ \\
\hline 2 h-Ins (mU/L) & $35.7(22.3-57.0)$ & $142.8 \pm 19.4^{* *}$ & 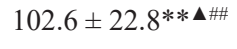 & $220.0 \pm 59.6^{* *}$ & $137.4 \pm 106.8^{* *}$ \\
\hline $\mathrm{TC}(\mathrm{mmol} / \mathrm{L})$ & $3.85 \pm 1.00$ & $4.46 \pm 1.03 * *$ & $4.57 \pm 0.85^{* *}$ & $4.35 \pm 0.75$ & $4.64 \pm 1.06^{* *}$ \\
\hline HDL-C $(\mathrm{mmol} / \mathrm{L})$ & $1.20 \pm 0.33$ & $1.26(1.14-1.48)^{*}$ & $1.31 \pm 0.38$ & $1.15 \pm 0.23$ & $1.17 \pm 0.35$ \\
\hline $\mathrm{TG}(\mathrm{mmol} / \mathrm{L})$ & $0.79(0.58-1.28)$ & $1.63 \pm 1.03 * *$ & $1.42 \pm 0.85^{*}$ & $2.37 \pm 1.18^{* * \boldsymbol{\Delta}}$ & $1.28 \pm 0.43^{*}$ \\
\hline LDL-C (mmol/L) & $2.17 \pm 0.85$ & $1.45(1.21-2.08)^{* *}$ & $1.61 \pm 0.66^{* *}$ & $2.22 \pm 0.87$ & $2.61 \pm 1.14^{\boldsymbol{\Lambda} \Lambda}$ \\
\hline $\mathrm{FFA}(\mu \mathrm{mol} / \mathrm{L})$ & $0.56 \pm 0.27$ & $0.66 \pm 0.24 *$ & $0.57 \pm 0.21$ & $0.58 \pm 0.17$ & $0.66(0.53-0.83)^{* *}$ \\
\hline SHBG (nmol/L) & $61.2 \pm 24.5$ & $29.1 \pm 12.0 * *$ & $57.2 \pm 27.7^{\boldsymbol{\Delta} \Delta \#}$ & $30.6 \pm 18.1 * *$ & $34.9 \pm 23.0 * *$ \\
\hline LH (IU/L) & $4.87 \pm 2.68$ & $10.3 \pm 5.7 * *$ & $10.3 \pm 7.7 * *$ & $7.92 \pm 5.75$ & $9.76 \pm 5.25^{* *}$ \\
\hline FSH (IU/L) & $8.07 \pm 1.92$ & $6.91 \pm 1.93^{* *}$ & $7.95 \pm 2.36^{\mathbf{\Delta}}$ & $8.33 \pm 2.58^{\Perp}$ & $7.29 \pm 1.83$ \\
\hline Test (nmol/L) & $1.80 \pm 0.73$ & $3.18 \pm 0.82 * *$ & $2.16 \pm 1.15^{\mathbf{\Delta} \triangle \#}$ & $3.32(2.76-3.99)^{* *}$ & $3.15 \pm 1.40 * *$ \\
\hline FAI & $2.56(1.81-4.89)$ & $13.26 \pm 5.91 * *$ & $4.12 \pm 1.89^{\mathbf{\Lambda}} \mathbf{\Delta} \#$ & $17.63 \pm 16.66^{* *}$ & $11.64 \pm 7.66^{* *}$ \\
\hline ZAG (10 ng/L) & $49.05(43.69-64.98)$ & $30.3 \pm 8.80 * *$ & 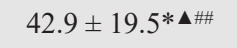 & $29.4 \pm 11.60 * *$ & $24.6 \pm 10.60 * *$ \\
\hline ZAG adjusted $^{\mathrm{a}}$ & $51.94 \pm 1.80$ & $31.12 \pm 2.78^{*}$ & $43.71 \pm 2.47 * \mathbf{\Delta} \#$ & $34.13 \pm 5.40^{*}$ & $24.21 \pm 3.41 * \boldsymbol{\Lambda}$ \\
\hline M-value $(\mathrm{mg} / \mathrm{min} / \mathrm{kg})$ & $10.14 \pm 2.55$ & $5.56 \pm 1.78 * *$ & $7.84 \pm 2.68 * * \mathbf{\Delta} \# \#$ & $3.79 \pm 1.49 * *$ & $5.64 \pm 2.37 * *$ \\
\hline HOMA-IR & $1.35(1.16-1.73)$ & $4.29 \pm 3.59^{* *}$ & $2.93 \pm 1.98 * * \#$ & $4.84 \pm 2.18^{* *}$ & $4.23 \pm 2.93 * *$ \\
\hline
\end{tabular}

PCOS, polycystic ovary syndrome; BMI, body mass index; WC, waist circumference; FAT (\%), body fat\%; SBP, systolic blood pressure; DBP, diastolic blood pressure; FBG, fasting blood glucose; 2 h-BG, 2 h post-glucose load blood glucose; Fins, fasting plasma insulin; 2 hIns, 2 h plasma insulin after glucose overload; TC, total cholesterol; HDL-C, high-density lipoprotein cholesterol; TG, triglyeride; LDL-C, low density lipoprotein cholesterol; FFA, free fatty acid; SHBG, sex hormone-binding globulin; LH, luteinizing hormone; FSH, follicular stimulating hormone; Test, testosterone; FAI, free androgen index; ZAG, Zinc- $\alpha 2$-Glycoprotein; M-value, whole body glucose uptake rate; HOMA-IR, HOMA-insulin resistance index. Values were given as mean $\pm \mathrm{SD}$ or median (interquartile Range). ${ }^{*} p<0.05, * * p<0.01 v s$. Controls (healthy women); $\boldsymbol{\Delta}_{p}<0.05, \mathbf{\Delta} \mathbf{\Delta} p<0.01 v s$. Classic group; ${ }^{\#} p<0.05$, \# $p<0.01 v s$. Ovulatory and or no-PCO morphology group. a, Means \pm standard error by general linear model with adjustment of BMI.

phenotypes were independently related to circulating ZAG levels in PCOS patients (Table 3, multivariable analysis).

In addition, thirty-six PCOS patients were diagnosed with MetS according to the International Diabetes Federation criteria (Table 4). In MetS subjects, increased waist circumference $(60 \%)$ and decreased HDL-C $(59 \%)$ contributed the most to the MetS diagnosis. The frequency of MetS diagnosis was higher in PCOS patients with the ovulatory phenotype $(64.2 \%)$, intermediate in patients with no-PCO morphology (36.8\%) and a classic phenotype $(36.0 \%)$, and lower in the normoandrogenic pheno- type (29.0\%) (Table 4).

\section{Discussion}

PCOS is a common endocrine disorder that includes a set of syndromes. However, the metabolic characteristics of PCOS and each phenotype are still controversial. The current study deployed state-of-the art methods to evaluate the circulating ZAG levels in PCOS patients with diverse phenotypes. In the current study, circulating ZAG levels of PCOS patients were considerably reduced relative to the healthy controls. PCOS patients, specifi- 
Table 2 Multiple regression analysis for the association between the different clinical elements used in diagnosis of PCOS and ZAG level.

\begin{tabular}{lccc}
\hline Feature & b Coefficient & SE & $p$ \\
\hline Model 1 & & & \\
Hyperandrogenism & -15.42 & 2.86 & $<\mathbf{0 . 0 0 1}$ \\
PCO morphology & 11.05 & 3.67 & $<\mathbf{0 . 0 1}$ \\
Oligoanovulation & 3.44 & 4.33 & 0.428 \\
Model 2 & & & \\
Hyperandrogenism & -6.05 & 2.91 & $<\mathbf{0 . 0 5}$ \\
PCO morphology & 5.95 & 3.09 & 0.057 \\
\hline Oligoanovulation & -6.20 & 3.55 & 0.084 \\
M value & 3.16 & 0.56 & $<\mathbf{0 . 0 0 1}$ \\
\hline HOMA-IR & -0.50 & 0.43 & 0.240 \\
\hline
\end{tabular}

ZAG was considered as a continuous variable. Statistically significant $p$ values are in bold. Model 1 includes only PCOSspecific clinical elements. Model 2 includes PCOS-specific clinical elements adjusted by M-Value and HOMA-IR. cally the ovulatory and no-PCO morphology phenotype subgroups, exhibited lower ZAG levels than those in the normoandrogenic subgroup, indicating that the ZAG protein most likely has potential androgen-specific activity and/or regulatory properties. In previous studies, lower serum ZAG levels were shown to be related to IR, T2DM, PCOS or obesity [12-14]. Kabil SL et al. recently showed reduced hepatic ZAG expression in diabetic rats maintained on a high-fat diet (HFD) [21]. Additionally, one study showed that the overexpression of ZAG alleviated HFD-induced IR and decreased the lipid contents of muscles in mice [22]. Another study showed that ZAG overexpression could protect against nonalcoholic fatty liver disease (NAFLD) in vivo or in vitro by ameliorating hepatic steatosis, IR, and inflammation [23]. Therefore, we speculated that decreased ZAG levels in obese and IR subjects potentially occur to offset IR-induced metabolic stress.

Based on the ESHRE/ASRM Rotterdam workshop and the AE-PCOS consensus statement, PCOS is considered a set of clinical syndromes with diverse phenotypes. Nonetheless, we observed that circulating ZAG levels were related to some diagnostic features of PCOS. We subsequently considered that circulating ZAG levels may

Table 3 Regression analysis for the association between the different phenotypes of PCOS and ZAG.

\begin{tabular}{|c|c|c|c|c|c|c|}
\hline \multirow{2}{*}{ PCOS phenotype $v s$. healthy women } & \multicolumn{3}{|c|}{ Univariable Analysis } & \multicolumn{3}{|c|}{ Multivariable Analysis } \\
\hline & b Coefficient & SE & $p$ & b Coefficient & SE & $p$ \\
\hline Classic & 3.593 & 0.648 & $<0.001$ & 1.960 & 0.716 & $<0.01$ \\
\hline Normoandrogenic & 1.655 & 0.439 & $<0.001$ & 0.837 & 0.542 & 0.122 \\
\hline Ovulatory & 2.495 & 0.693 & $<0.001$ & -0.324 & 0.906 & 0.720 \\
\hline no-PCO morphology & 3.335 & 0.785 & $<0.001$ & 1.869 & 0.880 & $<0.05$ \\
\hline M value & -0.586 & 0.080 & $<0.001$ & -0.453 & 0.103 & $<0.001$ \\
\hline HOMA-IR & 0.802 & 0.144 & $<0.001$ & 0.121 & 0.158 & 0.429 \\
\hline
\end{tabular}

ZAG was considered as a binary variable. Statistically significant $p$ values are in bold.

Table 4 Number (and percentage) of PCOS women with MetS and each components contributing to MetS diagnosis in the entire cohort of subjects and in women subdivided according to their PCOS phenotypes.

\begin{tabular}{lccccc}
\hline & \multirow{2}{*}{ All PCOS } & \multicolumn{4}{c}{ PCOS Phenotype } \\
\cline { 3 - 6 } & & Classic & Normoandrogenic & Ovulatory & no-PCO morphology \\
\hline MetS & $36(36.0)$ & $11(30.6)$ & $9(29.0)$ & $9(64.2)$ & $7(36.8)$ \\
$\mathrm{WC} \geq 80 \mathrm{~cm}$ & $60(60.0)$ & $23(63.8)$ & $15(48.4)$ & $10(71.4)$ & $12(63.2)$ \\
$\mathrm{FBG} \geq 5.6 \mathrm{mmol} / \mathrm{L}$ & $18(18.0)$ & $8(22.2)$ & $2(6.5)$ & $4(28.6)$ & $4(21.1)$ \\
$\mathrm{TG} \geq 1.70 \mathrm{mmol} / \mathrm{L}$ & $37(37.0)$ & $13(36.6)$ & $10(32.3)$ & $9(64.3)$ & $5(26.3)$ \\
$\mathrm{HDL}-\mathrm{C}<1.29 \mathrm{mmol} / \mathrm{L}$ & $59(59.0)$ & $20(55.6)$ & $14(45.2)$ & $11(78.6)$ & $14(73.7)$ \\
$\mathrm{BP} \geq 130 / 85 \mathrm{mmHg}$ & $25(21.0)$ & $5(13.9)$ & $7(22.6)$ & $7(50.0)$ & $6(31.6)$ \\
\hline
\end{tabular}

MetS, Metabolic syndrome; WC, waist circumference; FBG, fasting blood glucose; T2DM, type 2 diabetes mellitus; TG, triglyeride; HDLC, high-density lipoprotein cholesterol; BP, blood pressure. 
be a link between the metabolic features and reproductive disorders in PCOS patients. Our study addressed the following issues with reliable evidence: 1) whether circulating ZAG levels may be used as a biomarker for screening PCOS phenotypes and 2) whether circulating ZAG levels are related to certain metabolic characteristics of PCOS patients. Concerning the first issue, we found that circulating ZAG levels increased significantly in patients with normoandrogenic phenotypes but decreased significantly in patients with the no-PCO morphology phenotypes. Furthermore, circulating ZAG levels were linearly correlated with each phenotype. Therefore, our results indicate that circulating ZAG levels may be used as a biomarker for screening PCOS phenotypes. Concerning the second issue, to assess the metabolic characteristics of each phenotype, we used lipids, HOME-IR and EHC, which is the gold standard for diagnosing insulin resistance. In our study, HOMA-IR scores in the classic phenotype, the ovulatory phenotype and no-PCO morphology were significantly increased, indicating that the three phenotypes had insulin resistance. However, the normoandrogenic phenotype exhibited lower HOMA-IR scores and high M-value scores in this study, indicating IR is not a primary feature of the normoandrogenic phenotype. Therefore, we speculated that the normoandrogenic phenotype might have specific metabolic characteristics that require further study. These findings may also direct therapy for preventing complications related to metabolism in PCOS patients.

Our study boasts several key strengths, including 1) a population-based sample of young women; 2) accurate IR prediction by EHC, an IR evaluation gold standard; and 3) state-of-the-art methods deployed for characterizing PCOS patients. However, this study does reveal certain limitations. First, a few outlier instances, unavoidable in such a sample range, could adversely affect our results. Second, the cross-sectional design did not help clarify the correlation of circulating ZAG levels with other parameters. Additionally, a cross-sectional design could not possibly reflect variations in circulating ZAG over time; therefore, circulating ZAG levels should be assessed at each PCOS stage to investigate the role of ZAG in PCOS phenotypes.

\section{Conclusion}

Although IR and metabolic dysfunction are not associated with any diagnostic criteria in PCOS patients, some biomarkers, such as circulating ZAG levels, may guide physicians to identify the PCOS phenotype (such as the ovulatory or no-PCO morphology phenotypes). Instead of the insulin clamp, circulating ZAG levels may guide physicians to identify which PCOS patients should be screened for the risk of insulin resistance and metabolic complications. However, the normoandrogenic phenotype in PCOS patients may require a specific cohort from a metabolic standpoint.

\section{Acknowledgments}

We thank all of the participants of this study and the staff of the Department of Endocrinology, 9th People's Hospital of Chongqing, Chongqing, China, for their invaluable work. This study was funded by research grants from the Natural Science Foundation Project of Chongqing CSTC (cstc2017jcyjAX0269) and the Science and Technology key Program of Health Bureau of Chongqing (2016ZDXM039, 2017ZBXM018, 2019 QNXM020).

\section{Disclosure}

The authors declare that they have no competing interests.

\section{References}

1. Azziz R, Carmina E, Dewailly D, Diamanti-Kandarakis E, Escobar-Morreale HF, et al. (2009) The Androgen Excess and PCOS Society criteria for the polycystic ovary syndrome: the complete task force report. Fertil Steril 91: 456-488.

2. Randeva HS, Tan BK, Weickert MO, Lois K, Nestler JE, et al. (2012) Cardiometabolic aspects of the polycystic ovary syndrome. Endocr Rev 33: 812-841.

3. Reaven GM (2005) The insulin resistance syndrome: definition and dietary approaches to treatment. Annu Rev Nutr 25: 391-406.

4. Diamanti-Kandarakis E, Dunaif A (2012) Insulin resistance and the polycystic ovary syndrome revisited: an update on mechanisms and implications. Endocr Rev 33: 981-1030.

5. Carmina E, Lobo RA (2004) Use of fasting blood to assess the prevalence of insulin resistance in women with polycystic ovary syndrome. Fertil Steril 82: 661-665.

6. DeUgarte CM, Bartolucci A A, Azziz R (2005) Prevalence of insulin resistance in the polycystic ovary syndrome using the homeostasis model assessment. Fertil Steril 83: 1454-1460.

7. Kershaw EE, Flier JS (2004) Adipose tissue as an endocrine organ. J Clin Endocrinol Metab 89: 2548-2556.

8. Fantuzzi G (2005) Adipose tissue, adipokines, and inflammation. J Allergy Clin Immunol 115: 911-920. 
9. Bao Y, Bing C, Hunter L, Jenkins JR, Wabitsch M, et al. (2005) Zinc-alpha2-glycoprotein, a lipid mobilizing factor, is expressed and secreted by human (SGBS) adipocytes. FEBS Lett 579: 41-47.

10. Yeung DC, Lam KS, Wang Y, Tso AW, Xu A (2009) Serum zinc-alpha2-glycoprotein correlates with adiposity, triglycerides, and the key components of the metabolic syndrome in Chinese subjects. J Clin Endocrinol Metab 94: 2531-2536.

11. Eckardt K, Schober A, Platzbecker B, Mracek T, Bing C, et al. (2011) The adipokine zinc-alpha2-glycoprotein activates AMP kinase in human primary skeletal muscle cells. Arch Physiol Biochem 117: 88-93.

12. Yang M, Liu R, Li S, Luo Y, Zhang Y, et al. (2013) Zincalpha2-glycoprotein is associated with insulin resistance in humans and is regulated by hyperglycemia, hyperinsulinemia, or liraglutide administration: cross-sectional and interventional studies in normal subjects, insulin-resistant subjects, and subjects with newly diagnosed diabetes. Diabetes Care 36: 1074-1082.

13. Tian M, Liang Z, Liu R, Li K, Tan X, et al. (2016) Effects of sitagliptin on circulating zinc-alpha2-glycoprotein levels in newly diagnosed type 2 diabetes patients: a randomized trial. Eur J Endocrinol 174: 147-155.

14. Lai Y, Chen J, Li L, Yin J, He J, et al. (2016) Circulating Zinc-alpha2-glycoprotein levels and insulin resistance in polycystic ovary syndrome. Sci Rep 6: 25934.

15. Rotterdam ESHRE/ASRM-Sponsored PCOS Consensusn Workshop Group (2004) Revised 2003 consensus on diagnostic criteria and long-term health risks related to polycystic ovary syndrome. Fertil Steril 81: 19-25.

16. Alberti KG, Eckel RH, Grundy SM, Zimmet PZ, Cleeman JI, et al. (2009) Harmonizing the metabolic syndrome: a joint interim statement of the International Diabetes Fed- eration Task Force on Epidemiology and Prevention; National Heart, Lung, and Blood Institute; American Heart Association; World Heart Federation; International Atherosclerosis Society; and International Association for the Study of Obesity. Circulation 120: 1640-1645.

17. Luo $\mathrm{Y}, \mathrm{Li} \mathrm{L}, \mathrm{Xu} \mathrm{X}, \mathrm{Wu} \mathrm{T}$, Yang $\mathrm{M}$, et al. (2017) Decreased circulating BMP-9 levels in patients with Type 2 diabetes is a signature of insulin resistance. Clin Sci (Lond) 131: 239-246.

18. Jia Y, Yuan L, Hu W, Luo Y, Suo L, et al. (2014) Zincfinger BED domain-containing 3 (Zbed3) is a novel secreted protein associated with insulin resistance in humans. $J$ Intern Med 275: 522-533.

19. DeFronzo RA, Tobin JD, Andres R (1979) Glucose clamp technique: a method for quantifying insulin secretion and resistance. Am J Physiol 237: E214-E223.

20. Albareda M, Rodriguez-Espinosa J, Murugo M, de Leiva A, Corcoy R (2000) Assessment of insulin sensitivity and beta-cell function from measurements in the fasting state and during an oral glucose tolerance test. Diabetologia 43 : $1507-1511$.

21. Kabil SL, Mahmoud NM (2018) Canagliflozin protects against non-alcoholic steatohepatitis in type-2 diabetic rats through zinc alpha-2 glycoprotein up-regulation. Eur $J$ Pharmacol 828: 135-145.

22. Gao SX, Guo J, Fan GQ, Qiao Y, Zhao RQ, et al. (2018) ZAG alleviates HFD-induced insulin resistance accompanied with decreased lipid depot in skeletal muscle in mice. J Lipid Res 59: 2277-2286.

23. Xiao XH, Wang YD, Qi XY, Wang YY, Li JY, et al. (2018) Zinc alpha2 glycoprotein protects against obesityinduced hepatic steatosis. Int J Obes (Lond) 42: 14181430 . 